\title{
Supernova and neutron-star limits on large extra dimensions reexamined
}

\author{
Steen Hannestad \\ Department of Physics, University of Southern Denmark, Campusvej 55, 5230 Odense M, Denmark \\ and NORDITA, Blegdamsvej 17, 2100 Copenhagen, Denmark \\ Georg G. Raffelt \\ Max-Planck-Institut für Physik (Werner-Heisenberg-Institut), Föhringer Ring 6, 80805 München, Germany
}

(Dated: 3 April 2003)

\begin{abstract}
In theories with large extra dimensions, supernova (SN) cores are powerful sources of Kaluza-Klein (KK) gravitons. A large fraction of these massive particles are gravitationally retained by the newly born neutron star (NS). The subsequent slow KK decays produce potentially observable $\gamma$ rays and heat the NS. We here show that the back-absorption of the gravitationally trapped KK gravitons does not significantly change our previous limits. We calculate the graviton emission rate in a nuclear medium by combining the low-energy classical bremsstrahlung rate with detailed-balancing arguments. This approach reproduces the previous thermal emission rate, but it is much simpler and allows for a calculation of the absorption rate by a trivial phase-space transformation. We derive systematically the dependence of the SN and NS limits on the number of extra dimensions.
\end{abstract}

PACS numbers: PACS numbers: 11.10.Kk, 98.70.Vc, 12.10.-g

\section{INTRODUCTION}

Theories with large extra dimensions are a recent alternative to solve the hierarchy problem of particle physics 1, 2, 3, 4, 5, 6]. Within a certain class of models, the most restrictive limits on the size of the extra dimensions derive from the supernova (SN) emission of KaluzaKlein (KK) gravitons, particles with an essentially continuous spectrum of masses that are a generic feature of the new theory. Even though these new particles interact very weakly, i.e. the strength of ordinary gravitons, the number of modes and thus the size of the extra dimensions is constrained by the requirement that SN 1987A did not emit more KK gravitons than is compatible with the observed neutrino signal duration [7, 8, 9, 10].

In the simplest models, KK gravitons are stable except for their slow, gravitational-strength decay into photons, neutrinos, and other standard particles. Therefore, the decays of $\mathrm{KK}$ gravitons produced in all cosmic SNe will contribute to the measured cosmic $\gamma$-ray background, providing more restrictive limits than the SN 1987A energy-loss argument 11].

Long after the parent SN has exploded, a neutron star (NS) will continue to shine in $\gamma$-rays because a large fraction of the KK gravitons remains gravitationally trapped - most of them were produced with masses near the kinematical production threshold and thus with small velocities. Therefore, a NS is embedded in a halo of KK gravitons that shines in $100 \mathrm{MeV} \gamma$-rays. The EGRET non-observation of such signatures from nearby NSs thus provides restrictive limits [12]. The strongest constraints yet, however, are from avoiding excess heating of certain old NSs which otherwise could not cool to their observed low surface temperatures [12].

These arguments depend on the assumption that KK gravitons are not re-absorbed by inverse nuclear bremsstrahlung, a question that we failed to address when we raised our argument [12]. A crude estimate of the reabsorption effect gives a time scale larger than the ages of the stars that we used for our limits, but close enough to warrant a more detailed calculation. Moreover, if the re-absorption time scale is shorter than the KK decay lifetime, NSs will be heated directly by KK absorption rather than absorbing the decay products. The excess heat would increase and our bounds would improve.

The first goal of the present note is to calculate KK graviton absorption in a NS. Essentially this is done by phase-space transforming the emission rate. This exercise is particularly simple and transparent if one writes the graviton emission rate in a form that separates the response of the thermal nuclear medium from the phase space of the radiation. This approach significantly simplifies the original calculation of the emission rate of Hanhart, Phillips, Reddy and Savage [9] and illuminates the nature of the approximations made. To achieve the same level of precision we only need Weinberg's classical bremsstrahlung rate [13] together with the principle of detailed balancing. This approach closely follows previous calculations of neutrino or axion emission from a SN core based on the general properties of the thermal medium's response functions [14, 15]. Therefore, while the main goal of our derivation is to obtain the graviton absorption rate, its derivation is an illuminating exercise in its own right.

Our second goal is to extend our limits to the general case of $n$ extra dimensions. Previously, the limits on the compactification scale were explicitly stated only for the $n \leq 3$ cases. We use this opportunity to show how the various SN and NS limits scale with $n$.

In Sec. II we derive the emission and absorption of ordinary gravitons in the low-energy limit from a nuclear medium. In Sec. III we extend these results to the case of KK gravitons. In Sec. IV we revisit the SN and NS constraints, and in Sec. V we summarize our results. 


\section{NUCLEAR GRAVI-BREMSSTRAHLUNG}

\section{A. Differential Energy-Loss Rate}

The dominant graviton emission process from a SN core is nucleon-nucleon bremsstrahlung $N N \rightarrow N N g$ where for now $g$ stands for an ordinary graviton. In the limit of soft radiation the bremsstrahlung rate is determined by the measured nuclear scattering cross section alone, i.e. the nuclear scattering event and the associated bremsstrahlung process factorize [9]. In the soft limit the details of the scattering process do not matter, only the sudden change of the nucleons' energy-momentum tensor is responsible for the radiation.

Assuming that two non-relativistic nucleons collide with the initial CM momenta $\mathbf{p}_{i}=\mathbf{p}_{1}=-\mathbf{p}_{2}$ to the final states $\mathbf{p}_{f}=\mathbf{p}_{3}=-\mathbf{p}_{4}$, the differential amount of emitted gravi-bremsstrahlung energy is [13]

$$
\frac{d E_{g}}{d \omega}=\frac{16 G_{\mathrm{N}}}{5 \pi} \frac{\mathbf{p}_{i}^{4}+\mathbf{p}_{f}^{4}-2\left(\mathbf{p}_{i} \cdot \mathbf{p}_{f}\right)^{2}}{M^{2}},
$$

where $G_{\mathrm{N}}$ is Newton's constant, $M$ the nucleon mass, and $\omega$ the graviton energy. We may also write this in terms of the total initial and final kinetic nucleon energies, $E_{i}=$ $\mathbf{p}_{1}^{2} / 2 M+\mathbf{p}_{2}^{2} / 2 M=\mathbf{p}_{i}^{2} / M$ and $E_{f}=\mathbf{p}_{3}^{2} / 2 M+\mathbf{p}_{4}^{2} / 2 M=$ $\mathbf{p}_{f}^{2} / M$, and in terms of the CM scattering angle as

$$
\frac{d E_{g}}{d \omega}=\frac{16 G_{\mathrm{N}}}{5 \pi}\left(E_{i}^{2}+E_{f}^{2}-2 E_{i} E_{f} \cos ^{2} \Theta_{\mathrm{CM}}\right) .
$$

Energy conservation implies $E_{f}=E_{i}-\omega$ so that

$$
\frac{d E_{g}}{d \omega}=\frac{8 G_{\mathrm{N}}}{5 \pi}\left(E_{i}+E_{f}\right)^{2} \sin ^{2} \Theta_{\mathrm{CM}}+\mathcal{O}\left(\omega^{2}\right),
$$

an approximation used in Ref. [9].

If the emission of soft gravitons $(\omega \rightarrow 0)$ is viewed as a classical process, i.e. the colliding nucleons as external sources, energy conservation does not apply, $E_{f}=E_{i}$, and we get the usual flat bremsstrahlung spectrum,

$$
\frac{d E_{g}}{d \omega}=\frac{32 G_{\mathrm{N}}}{5 \pi} E_{i}^{2} \sin ^{2} \Theta_{\mathrm{CM}} .
$$

Augmenting this classical result with detailed balancing will give us the correct emission rate up to $\mathcal{O}(\omega)$ as in the more complicated treatment of Ref. [9].

Next, we introduce the nucleon scattering amplitude $\mathcal{A}$ which is normalized such that the differential cross section for neutron-neutron scattering is

$$
\frac{d \sigma}{d \Omega}=\frac{M^{2} \pi^{4}|\mathcal{A}|^{2}}{(2 \pi)^{6}}
$$

Note that $|\mathcal{A}|^{2}$ includes a spin sum over initial- and finalstate neutrons while $d \sigma / d \Omega$ is averaged over initial and summed over final spins. If one takes $|\mathcal{A}|^{2}$ to be independent of scattering angles, the total cross section is

$$
\sigma=\frac{M^{2}|\mathcal{A}|^{2}}{32 \pi}=25 \mathrm{mb} \text {. }
$$

A factor $\frac{1}{2}$ for identical final-state nucleons was included and the numerical value was quoted in Ref. [9] as a good approximation for the conditions of interest.

With these ingredients the differential energy-loss rate of a neutron medium in the soft limit is

$$
\left.\frac{d Q}{d \omega}\right|_{\omega=0}=\int d \Gamma S|\mathcal{A}|^{2}\left[\frac{32 G_{\mathrm{N}}}{5 \pi} E_{i}^{2} \sin ^{2} \Theta_{\mathrm{CM}}\right]
$$

where $S=\frac{1}{4}$ is a statistics factor for identical particles in the initial and final state while $d \Gamma$ symbolizes the neutron phase space integration, including all thermal occupation numbers, blocking factors and the energy-momentum $\delta$ function. True to our soft-radiation approximation, the graviton energy-momentum does not appear in this $\delta$ function. Without the term in square brackets Eq. (7) is the rate of $n n$ collisions per unit volume.

We now read off the dependence of the energy-loss rate $Q$ on various input parameters. If the medium is nondegenerate, the $n n$ collision rate per unit volume is the neutron number density squared times the cross section times an average thermal relative velocity, the latter being proportional to $(T / M)^{1 / 2}$ with $T$ the temperature. Further, from the bremsstrahlung rate we have an average $E_{i}^{2}$ which is proportional to $T^{2}$. A typical emitted graviton energy is of order $T$ so that finally

$$
Q \propto G_{\mathrm{N}} \sigma n_{B}^{2} T^{7 / 2} M^{-1 / 2},
$$

where $n_{B}$ is the density of baryons (here neutrons).

We will perform explicit calculations only for the case of non-relativistic non-degenerate neutrons. In that case the phase-space integral can be transformed to one over CM momenta [9]. Integrating a quantity $F$ over the nucleon phase space yields

$$
\begin{aligned}
\int d \Gamma F & =n_{B}^{2}\left(\frac{M}{\pi}\right)^{3 / 2} \frac{T^{1 / 2}}{4} \\
& \times \int_{0}^{\infty} d u_{i} \int_{0}^{\infty} d u_{f}\left(u_{i} u_{f}\right)^{1 / 2} e^{-u_{i}} \delta\left(u_{i}-u_{f}\right) \\
& \times \frac{1}{2} \int_{-1}^{+1} d \cos \Theta_{\mathrm{CM}} F
\end{aligned}
$$

where $u_{i, f}=E_{i, f} / T$. We have explicitly kept the energy $\delta$ function for later convenience.

Applying this result first to the $n n$ scattering rate per unit volume we insert $F=S|\mathcal{A}|^{2}$. Assuming that the scattering amplitude is independent of the CM energy and the scattering angle we find explicitly

$$
\Gamma_{n n}=\sigma n_{B}^{2}\left(\frac{4 T}{\pi M}\right)^{1 / 2}=\sigma \frac{n_{B}^{2}}{2}\left\langle v_{\mathrm{rel}}\right\rangle
$$

where the factor $\frac{1}{2}$ accounts for identical initial-state particles. The corresponding final-state factor is already included in the total cross section. 
Next we perform the analogous integral for the differential energy-loss rate and find

$$
\begin{aligned}
\left.\frac{d Q}{d \omega}\right|_{\omega=0} & =\Gamma_{n n} \frac{128}{5 \pi} G_{\mathrm{N}} T^{2} \\
& =\frac{256}{5 \pi^{3 / 2}} \frac{G_{\mathrm{N}} \sigma n_{B}^{2} T^{5 / 2}}{M^{1 / 2}}
\end{aligned}
$$

for the "classical" bremsstrahlung losses of the medium.

\section{B. Total Rate from Detailed Balancing}

The differential rate derived thus far is not yet useful for calculating the total $Q$. To this end we observe that the emission, absorption, or scattering of any radiation by any medium is described by a suitable dynamical structure function. This is a quantum correlator of those medium operators which couple to the relevant radiation. For example, for neutrino pair emission, pair absorption, or scattering, we need the nucleon spin correlator because neutrinos couple primarily to the nucleon spin [14, 15]. For graviton emission we need a dynamical structure function describing the microscopic fluctuations of the medium's energy-momentum tensor.

However, in the soft limit when the energy transfer $\omega$ is small we may use the long-wavelength limit where the radiation's momentum transfer is ignored. The energyloss rate of any type of radiation is then of the form

$$
Q=\int \frac{d^{3} \mathbf{k}}{2 \omega(2 \pi)^{3}} \omega S(-\omega)
$$

where $S(\omega)$ is a function of the energy transfer alone. Apart from overall coefficients, $S$ is the dynamical structure function in the long-wavelength limit. The integral is over the invariant phase-space of the radiation, one factor $\omega$ accounts for the energy carried by the radiation. Note that from the medium's perspective a negative $\omega$ is energy lost, a positive $\omega$ energy gained. Therefore, bremsstrahlung emission involves $S(-\omega)$.

The only property of relevance to our present discussion is the detailed-balancing requirement

$$
S(-\omega)=S(\omega) e^{-\omega / T}
$$

which is a general consequence of the non-commuting nature of the correlated quantum operators describing the thermal medium, i.e. it strictly is a quantum effect. This condition ensures that the efficiency of emission and absorption of radiation is such that in equilibrium the radiation will reach a thermal distribution function. As an immediate consequence the most general structure function is of the form

$$
S(\omega)=\bar{S}(\omega) \frac{2}{1+e^{-\omega / T}}
$$

where $\bar{S}(\omega)=\frac{1}{2}[S(\omega)+S(-\omega)]$ is symmetric in $\omega$.
From Eq. (12) we may now write the differential energy-loss rate in the form

$$
\frac{d Q}{d \omega}=\frac{\omega^{2}}{(2 \pi)^{2}} \bar{S}(\omega) \frac{2}{1+e^{\omega / T}} .
$$

Comparing with Eq. (11) we recognize that we may write

$$
\bar{S}(\omega)=\frac{S_{0}}{\omega^{2}} s(\omega / T)
$$

where $s(x)$ is a dimensionless even function normalized to $s(0)=1$ and

$$
\begin{aligned}
S_{0} & =\frac{1024 \pi^{1 / 2}}{5} \frac{G_{\mathrm{N}} \sigma n_{B}^{2} T^{5 / 2}}{M^{1 / 2}} \\
& =1.547 \times 10^{16} \mathrm{erg} \mathrm{cm}^{-3} \mathrm{~s}^{-1} \mathrm{MeV}^{-1} T_{30}^{5 / 2} \rho_{3}^{2} .
\end{aligned}
$$

Here, $T_{30}=T / 30 \mathrm{MeV}$ and $\rho_{3}=\rho / 3 \times 10^{14} \mathrm{~g} \mathrm{~cm}^{-3}$. Since $S(\omega)$ is even apart from the "trivial" detailedbalancing factor, we obtain the full differential energyloss rate up to first order in $x=\omega / T$ even though we have calculated the bremsstrahlung rate only to zeroth order in $\omega / T$.

Our approach of using the concept of the structure function kills two birds with one stone. We obtain the differential emission rate up to order $\omega / T$ on the basis of the strictly classical soft-radiation bremsstrahlung rate. Second, the inverse process, graviton absorption, is simply a different phase-space integral over the same structure function and thus trivial to extract.

A calculation up to $\mathcal{O}(\omega / T)$ is the best one can do in the framework of the soft-radiation approximation. A more accurate calculation would require modeling the nucleon interaction in detail because the scattering and bremsstrahlung parts of the process no longer factorize. Of course, a more precise calculation would also require taking many-body effects into account, i.e. one would need to calculate properly the dynamical structure function, an impossible task at present.

In the absence of any more precise insights concerning the behavior of the structure function we may use the simplest approximation compatible with our level of approximation and take $s(x)=1$, dropping all higher-order terms. In this case we find for the total emission rate

$$
Q=\frac{512 \ln 2}{5 \pi^{3 / 2}} G_{\mathrm{N}} \sigma n_{B}^{2} T^{7 / 2} M^{-1 / 2}
$$

The coefficient is numerically 12.75 .

The corresponding results of Ref. [9] are recovered if in the nuclear phase-space integral Eq. (9) we include the graviton energy, i.e. energy conservation now reads $\delta\left(u_{i}-u_{f}-x\right)$ with $x=\omega / T$. Moreover, we must include the expression Eq. (3) for $d E_{g} / d \omega$. One first performs $\int d \omega$ to remove the $\delta$ function and then integrates the remaining expression. The coefficient of $Q$ is then found to be $47104 /\left(525 \pi^{3 / 2}\right) \approx 16.11$, somewhat larger than our result. 
We can also extract the structure function implied by this treatment. To this end we leave the $\int d \omega$ integration open and rather do $\int d u_{i}$ to remove the $\delta$ function. Collecting all factors we find

$$
\begin{aligned}
s(x) & =\frac{1+e^{-x}}{48} \int_{0}^{\infty} d u_{f}\left(u_{f}^{2}+u_{f} x\right)^{1 / 2} e^{-u_{f}}\left(2 u_{f}+x\right)^{2} \\
& =1+\frac{5}{48} x^{2}+\mathcal{O}\left(x^{4}\right) .
\end{aligned}
$$

However, $s(x)$ is not analytic, the coefficient of the $x^{4}$ term diverges. This expression indeed agrees with ours up to $\mathcal{O}(x)$. Put another way, up to $\mathcal{O}(\omega)$ Ref. [9] explicitly recovers the detailed-balancing condition that we used as input information.

We can go through the same exercise for $d E_{g} / d \omega$ of Eq. (2) and find $s(x)=1+(5 / 16) x^{2}+\mathcal{O}\left(x^{4}\right)$. This again deviates from the other results only at $\mathcal{O}\left(x^{2}\right)$.

In summary, even though one has used the softradiation approximation which is valid only for $\omega \ll T$, one obtains an estimate of the emission rate which likely is correct within a numerical factor of order 1. Still, this approach has essentially the status of an "educated dimensional analysis." A real calculation would require knowledge of the relevant dynamical structure function of the nuclear medium, not just its long-wavelength, softradiation limit.

\section{Inverse Bremsstrahlung}

We are now in a position to calculate the graviton absorption rate by a nuclear medium. For simplicity we may start with the energy-loss rate Eq. (12), leave out the graviton phase space integration except for the factor $1 / 2 \omega$, remove the factor $\omega$ which represents the emitted energy, include a factor $1 / 2$ because the energy-loss rate is summed over two graviton polarizations, and substitute $\omega \rightarrow-\omega$ in the structure function because the medium now gains energy. Then we find

$$
\begin{aligned}
\Gamma_{g}(\omega) & =\frac{S(\omega)}{4 \omega} \\
& =\frac{256 \pi^{1 / 2}}{5} \frac{G_{N} \sigma \rho^{2} T^{5 / 2}}{M^{5 / 2}} \frac{2}{1+e^{-\omega / T}} \frac{1}{\omega^{3}} \\
& =3.7 \times 10^{-17} \mathrm{~s}^{-1} \rho_{3}^{2} T_{30}^{5 / 2} \omega_{100}^{-3}
\end{aligned}
$$

where $\rho_{3}=\rho / 3 \times 10^{14} \mathrm{~g} \mathrm{~cm}^{-3}, T_{30}=T / 30 \mathrm{MeV}$, and $\omega_{100}=\omega / 100 \mathrm{MeV}$. We have assumed $s(x)=1$ and in the numerical expression also $e^{-\omega / T}=0$.

\section{KALUZA-KLEIN GRAVITONS}

\section{A. Large Extra Dimensions}

Next we assume that space has $n$ large extra dimensions in which gravity can propagate. String theory suggests a total of 11 space-time dimensions so that there are at most 7 large extra dimensions in addition to our 4 -dimensional space-time. Therefore, we will limit our explicit calculations and results to $1 \leq n \leq 7$.

We further assume that the extra dimensions are toroidally compactified with an equal radius $R$ for all of them. Put another way, the linear size of each extra dimension is $2 \pi R$ and the volume of the compactified space is $V_{n}=(2 \pi R)^{n}$.

Kaluza-Klein (KK) gravitons that propagate in the extra dimensions with a momentum $p$ will appear to us as having a mass $m=p$. The modes are discrete with a density of states $V_{n} /(2 \pi)^{n}=R^{n}$. A summation over all modes in the extra dimensions corresponds to summing over all masses of KK gravitons in our world. For large extra dimensions the modes are narrowly spaced so that the sum over masses transforms to an integral

$$
\sum_{\text {modes }} \rightarrow \Omega_{n} R^{n} \int d m m^{n-1}
$$

where

$$
\Omega_{n}=\frac{2 \pi^{n / 2}}{\Gamma(n / 2)}
$$

is the surface of the $n$-dimensional unit sphere.

Following the conventions of Ref. [6] we note that the fundamental scale of the new theory is the effective Planck scale $\bar{M}_{4+n}$ of the higher dimensional space. It relates to the conventional Planck scale $M_{\mathrm{Pl}}=G_{\mathrm{N}}^{-1 / 2}=$ $1.22 \times 10^{19} \mathrm{GeV}$ by

$$
\bar{M}_{\mathrm{Pl}}^{2}=V_{n} \bar{M}_{4+n}^{2+n}
$$

where $\bar{M}_{\mathrm{Pl}}=M_{\mathrm{Pl}} / \sqrt{8 \pi}$. Numerically we find

$$
\frac{\bar{M}_{4+n}}{1 \mathrm{TeV}}=\left(5.922 \times 10^{30}\right)^{\frac{1}{2+n}}\left(\frac{3.140 \times 10^{-20} \mathrm{~m}}{R}\right)^{\frac{n}{2+n}}
$$

for the relationship between $\bar{M}_{4+n}$ and $R$.

Note that $\bar{M}_{4+n}$ is different from the energy scale $M_{4+n}$ that was used by Hanhart et al. 99, 10] and that we called $M=M_{4+n}$ in our previous papers [11, 12]. This energy scale was defined from the relation

$$
4 \pi G_{\mathrm{N}}=\left(\frac{1}{R M}\right)^{n} \frac{1}{M^{2}} .
$$

With Eq. (23) this implies

$$
M=2^{1 /(n+2)}(2 \pi)^{n /(n+2)} \bar{M}_{4+n} .
$$

This relationship allows one to translate our new limits on $\bar{M}_{4+n}$ into limits on the previous parameter $M$. In the second row of Table VI we give explicit values for $M / \bar{M}_{4+n}$, i.e. one should multiply our new limits with this factor to obtain the corresponding limits on the old parameter $M$. 


\section{B. Absorption and Emission of KK Gravitons}

For KK gravitons, the absorption rate will be similar to that for ordinary gravitons of the same energy. Therefore, we may use Eq. (20) directly for this purpose.

For the energy-loss rate we must take account of the large number of available modes. For a fixed KK mode with mass $m$ one has to include in Eq. (12) a factor [9]

$$
\left(\frac{19}{18}+\frac{11}{9} \frac{m^{2}}{\omega^{2}}+\frac{2}{9} \frac{m^{4}}{\omega^{4}}\right)
$$

that already includes a summation over polarization states. In addition, $\int d^{3} \mathbf{k} \rightarrow 4 \pi \int \omega k d \omega$ with $k=$ $\left(\omega^{2}-m^{2}\right)^{1 / 2}$. The total emission rate then obtains by summing over all masses. With Eq. (21) one thus finds

$$
Q_{n}=\frac{\Omega_{n} R^{n}}{(2 \pi)^{2}} \int_{0}^{\infty} d \omega S(-\omega) \omega^{n+1} \int_{0}^{\omega} d m G_{n-1}\left(\frac{m}{\omega}\right)
$$

where we have defined

$$
G_{p}(\mu)=\mu^{p}\left(1-\mu^{2}\right)^{1 / 2}\left(\frac{19}{18}+\frac{11}{9} \mu^{2}+\frac{2}{9} \mu^{4}\right),
$$

using $\mu=m / \omega$. Performing the integral over masses explicitly yields

$$
Q_{n}=\frac{g_{n} R^{n}}{(2 \pi)^{2}} \int_{0}^{\infty} d \omega \omega^{n+2} S(-\omega)
$$

where

$$
g_{n}=\Omega_{n} \int_{0}^{1} d \mu G_{n-1}(\mu)=\frac{5 \pi^{\frac{n+1}{2}}}{48} \frac{19+18 n+3 n^{2}}{\Gamma\left(\frac{7+n}{2}\right)} .
$$

Note that $g_{0}=19 / 18$ does not represent the emission of ordinary gravitons because of differences in the summation over polarization states.

With these results it is straightforward to state the differential energy-loss rate. We read from Eq. (30)

$$
\frac{d Q_{n}}{d \omega}=\frac{S_{0}}{(2 \pi)^{2}} g_{n}(R T)^{n}\left(\frac{\omega}{T}\right)^{n} \frac{2}{1+e^{\omega / T}} s(\omega / T) .
$$

Likewise

$$
\frac{d Q_{n}}{d m}=\frac{S_{0}}{(2 \pi)^{2}} \Omega_{n}(R T)^{n}\left(\frac{m}{T}\right)^{n-1} F(m / T),
$$

where

$$
F(y)=\int_{y}^{\infty} d x \frac{2}{1+e^{x}} s(x) G_{0}\left(\frac{y}{x}\right)
$$

with $y=m / T$ and $x=\omega / T$. For $s(x)=1$ a simple approximation is $F(y) \approx 2 y^{0.11} e^{-1.045 y}$ that is good to about $\pm 7 \%$ for $0.1<\mu<20$.

If we finally use our expression for the structure function with the simplest assumption $s(x)=1$ we find

$$
Q_{n}=g_{n} f_{n}(R T)^{n} Q_{0}
$$

where

$$
\begin{aligned}
Q_{0} & =\frac{512}{5 \pi^{3 / 2}} G_{\mathrm{N}} \sigma n_{B}^{2} T^{7 / 2} M^{-1 / 2} \\
& =2.35 \times 10^{16} \mathrm{erg} \mathrm{cm}^{-3} \mathrm{~s}^{-1} T_{30}^{7 / 2} \rho_{3}^{2} .
\end{aligned}
$$

Here, $T_{30}=T / 30 \mathrm{MeV}$ and $\rho_{3}=\rho / 3 \times 10^{14} \mathrm{~g} \mathrm{~cm}^{-3}$ and

$$
f_{n}=\left(1-2^{-n}\right) \Gamma(n+1) \zeta(n+1),
$$

valid for $n \geq 1$.

In Table $\llbracket$ we give numerical values for $\left(g_{n} f_{n}\right)^{1 / n}$ because this is the effective $n$-dependent coefficient of $R$ in the emission-rate formula. A simple approximation is

$$
\left(g_{n} f_{n}\right)^{1 / n} \approx 1.81 n^{0.425}
$$

good to better than $1 \%$ in the range $1 \leq n \leq 10$.

The corresponding emission rate of Ref. [9], their Eq. (56), is systematically larger by factors of up to a few because their $s(x)$ grows quadratically with $x$, increasing the emission of higher-energy gravitons. Recall that for the emission rate one needs the structure function for $\omega / T$ of a few while the soft-radiation approximation is only valid for $\omega \ll T$. Therefore, our result agrees with Ref. [9] up to the claimed accuracy of either result. The soft-radiation calculation can not predict the emission rate to better than a factor of a few.

It is straightforward to calculate the average values of several parameters of the emitted gravitons. In Table I we give explicit numerical values for the average energy $\langle\omega\rangle$, the average mass $\langle m\rangle$, and the average velocity $\langle v\rangle$. We also give the retention fraction $f_{\text {trap }}$ in a typical neutron star and the average mass of the trapped gravitons as explained in the following Section.

TABLE I: Various coefficients described in the text.

\begin{tabular}{lll}
\hline \hline$n$ & $\left(g_{n} f_{n}\right)^{1 / n}$ & $\left(\Omega_{n} h_{n}\right)^{1 / n}$ \\
1 & 1.7943 & 0.3763 \\
2 & 2.4544 & 0.9731 \\
3 & 2.8981 & 1.1607 \\
4 & 3.2597 & 1.2001 \\
5 & 3.5771 & 1.1891 \\
6 & 3.8662 & 1.1605 \\
7 & 4.1348 & 1.1263 \\
\hline \hline
\end{tabular}

TABLE II: Average properties of the emitted KK gravitons.

\begin{tabular}{llllll}
\hline \hline$n$ & $\langle\omega\rangle / T$ & $\langle m\rangle / T$ & $\langle v\rangle$ & $f_{\text {trap }}$ & $\langle m\rangle_{\text {trap }} / T$ \\
1 & 1.1866 & 0.5784 & 0.8061 & 0.0963 & 1.0902 \\
2 & 2.1923 & 1.4054 & 0.7052 & 0.1816 & 2.0176 \\
3 & 3.1514 & 2.2663 & 0.6384 & 0.2607 & 2.9052 \\
4 & 4.1060 & 3.1516 & 0.5892 & 0.3342 & 3.7917 \\
5 & 5.0691 & 4.0601 & 0.5506 & 0.4020 & 4.6890 \\
6 & 6.0429 & 4.9888 & 0.5191 & 0.4643 & 5.5993 \\
7 & 7.0257 & 5.9335 & 0.4927 & 0.5211 & 6.5208 \\
\hline \hline
\end{tabular}




\section{Neutron-Star Retention of KK Gravitons}

The KK gravitons are produced with barely relativistic velocities. Therefore, the SN core will retain a large fraction of them gravitationally after thermal production by the nuclear medium. In order to estimate the retention fraction we take the NS to be a homogeneous sphere with a density $3 \times 10^{14} \mathrm{~g} \mathrm{~cm}^{-3}$ and a mass $M_{\mathrm{NS}}=1.4 M_{\odot}$, corresponding to a radius of $R_{\mathrm{NS}}=13.06 \mathrm{~km}$ and a surface gravitational potential of

$$
U_{\mathrm{NS}}=-\frac{G_{\mathrm{N}} M_{\mathrm{NS}}}{R_{\mathrm{NS}}}=-0.1139 .
$$

We here use Newtonian physics rather than general relativity. Our simple NS model leads to a radial dependence of the gravitational potential within the NS of

$$
U(r)=U_{\mathrm{NS}} \frac{3-r^{2}}{2},
$$

where $r$ is a dimensionless radial coordinate that varies from 0 to 1 in the NS. A KK graviton produced at radius $r$ with energy $\omega$ remains trapped if its kinetic plus gravitational energy is negative, $U(r)+\frac{1}{2} v^{2}<0$ or

$$
\omega \geq m \geq m_{\text {trap }}(r)=\omega[1+U(r)],
$$

where we have used that $|U| \ll 1$.

The graviton number emissivity is given by Eq. (30) if we drop one power of $\omega$ under the integral. The number emissivity of those KK gravitons that remain trapped, averaged over the entire $\mathrm{NS}$, is found by replacing $g_{n}$ with $g_{n}^{\text {trap }}$ in these expressions. The latter is found if in Eq. (31) we substitute

$$
\int_{0}^{1} d \mu \ldots \rightarrow 3 \int_{0}^{1} d r r^{2} \int_{\mu_{\text {trap }}(r)}^{1} d \mu \ldots
$$

where $\mu_{\text {trap }}=m_{\text {trap }} / \omega=1+U(r)$.

For fixed $n$ the distribution of emitted masses is a universal function of the parameter $\mu=m / \omega$, independently of the energy $\omega$. The velocity of a KK graviton is $v=\left(1-\mu^{2}\right)^{1 / 2}$ and thus only a function of $\mu$ so that the distribution of KK velocities is also independent of their energy. Therefore, the trapping fraction is independent of the KK energy, in every energy class $\omega$ the same fraction is retained given by $g_{n}^{\text {trap }} / g_{n}$. Explicit trapping fractions for different values of $n$ are shown in Table II

As another consequence the average energy of the trapped KK gravitons is identical with that of the escaping ones. However, the average mass of those that remain trapped is much larger than the overall average. We give explicit results for $\langle m\rangle_{\text {trap }}$ in Table [I] These values are only slightly smaller than $\langle\omega\rangle$, reflecting the fact that the trapped particles are slow-moving and thus have only small amounts of kinetic energy.

\section{CONSTRAINTS ON LARGE EXTRA DIMENSIONS}

\section{A. Supernova 1987A}

We begin with the classical SN 1987A energy-loss limit on the emission of $\mathrm{KK}$ gravitons [7, 8, 9, 10]. The duration of the SN 1987A neutrino signal precludes that too much energy was emitted in an invisible channel. For several cases involving axions or right-handed neutrinos, self-consistent cooling calculations were performed to determine the relationship between the allowed coupling strength of the exotic particles and the duration of the neutrino burst. The result can be summarized by the simple criterion that the exotic energy-loss rate of a nuclear medium at a density of $3 \times 10^{14} \mathrm{~g} \mathrm{~cm}^{-3}$ and a temperature of $30 \mathrm{MeV}$ should not exceed $10^{19} \mathrm{erg} \mathrm{g}^{-1} \mathrm{~s}^{-1}$ [16, 17]. This criterion is not crude or arbitrary, but is calibrated by numerical simulations of different authors with different codes and for different cases and reproduces these results surprisingly well. The numerical studies of Ref. [10] for the specific case of KK gravitons confirms once more the accuracy of the simple criterion.

We have only considered a non-degenerate neutron medium. For the realistic case of a mixture of protons and neutrons the emission rate would be somewhat larger, while degeneracy effects would slightly decrease it. The soft-radiation calculation is only accurate to within a factor of a few. Therefore, it is not worth worrying about the exact influence of the mild nucleon degeneracy or the chemical composition. The simple energy-loss criterion together with Eq. (35) thus implies

$$
R \lesssim 6.58 \times 10^{-15} \mathrm{~m}\left(1.28 \times 10^{17}\right)^{1 / n}\left(g_{n} f_{n}\right)^{-1 / n}
$$

We give the explicit limits for $1 \leq n \leq 7$ in Table $\nabla$ The corresponding limits on $\bar{M}_{4+n}$ are given in Table $\nabla \mathbf{~}$

For $n=2$ our limit $R \lesssim 0.96 \mu \mathrm{m}$ compares with $0.70 \mu \mathrm{m}$ of Ref. [9] and $0.66 \mu \mathrm{m}$ of Ref. [10]. For $n=3$ our limit $R \lesssim 1.14 \mathrm{~nm}$ compares with $0.83 \mathrm{~nm}$ of Ref. 9] and $0.8 \mathrm{~nm}$ of Ref. [10]. The small differences are perfectly in line with the different approximation made in the calculation of the emission rate and with our using the simple energy-loss criterion rather than a specific numerical simulation as in Ref. [10].

\section{B. All Cosmic Supernovae}

In the toroidal compactification scheme assumed in our discussion the only decay channels for KK gravitons are into those standard-model particles that are kinematically allowed, notably into $2 \gamma, e^{-} e^{+}$and $\nu \bar{\nu}$. The coupling is of gravitational strength so that the mean lifetime is very large [18]

$$
\tau_{2 \gamma}=\frac{1}{2} \tau_{e^{-} e^{+}}=\tau_{\nu \bar{\nu}} \simeq 6 \times 10^{9} \mathrm{yr}\left(\frac{100 \mathrm{MeV}}{m}\right)^{3} .
$$


This is comparable to the age of the universe for masses in the $100 \mathrm{MeV}$ range that would be typical for those $\mathrm{KK}$ gravitons emitted by a $\mathrm{SN}$ core.

The photons from these decays from all past SNe would contribute to the cosmic $\gamma$-ray background as measured by the EGRET instrument 19], constraining the amount of KK gravitons that may have been emitted by all cosmic SNe 11. The measured diffuse $\gamma$ background falls with energy roughly as $E^{-2}$. We have explained in Ref. 11] that this particular power-law behavior implies that the limit on the KK emission by SNe is nearly independent of the assumed core temperature and nearly independent of the KK mass spectrum as long as the masses are so large that most of the gravitons would have decayed within a Hubble time. This is the case for $T \gtrsim 20 \mathrm{MeV}$ and $n \gtrsim 2$.

In Ref. 11] we found that the EGRET data imply that a typical SN core must not lose more than about $0.5 \%$ of its energy in KK gravitons. With a realistic SN rate the limit would be more restrictive by factors between 10 and 100. In the spirit of deriving conservative limits it is thus justified to assume that for all $n$ not more than $1 \%$ of the energy loss is allowed that we used for the SN 1987A limit. Put another way, the SN 1987A limits on $R$ scale with $\left(10^{-2}\right)^{1 / n}$. The corresponding explicit limits for $1 \leq$ $n \leq 7$ are given in Tables $\nabla$ and $\nabla]$ respectively.

\section{Supernova Remnant Cas A}

The SN remnant Cas A probably corresponds to Flamsteed's SN of 1680, but in any case is so young that the cloud of emitted KK gravitons would still appear as a point source to EGRET, even ignoring the fact that a large fraction of them is gravitationally retained [11]. The absence of an EGRET source at the location of Cas A implies that the flux of decay photons at Earth from this source is limited by

$$
\Phi_{E_{\gamma}>100 \mathrm{MeV}} \lesssim 10^{-7} \mathrm{~cm}^{-2} \mathrm{~s}^{-1} .
$$

In order to predict the photon flux at Earth we begin with the KK emissivity Eq. (28). To get the number flux we must include a factor $\omega^{-1}$ under the integral. Further, we must include the decay rate $\Gamma_{2 \gamma}$, involving the timedilation factor $m / \omega$ and $\tau_{2 \gamma}^{-1}$ from Eq. (44), i.e.

$$
\Gamma_{2 \gamma}=\gamma \frac{m^{4}}{\omega}
$$

with $\gamma=5.3 \times 10^{-24} \mathrm{~s}^{-1} \mathrm{MeV}^{-3}$. We further need to multiply with the volume $V_{\mathrm{NS}}$ of the NS and with the time-scale $\Delta t_{\mathrm{NS}}$ of $\mathrm{KK}$ emission, i.e. the cooling time scale of the nascent neutron star. We will use $V_{\mathrm{NS}}=M_{\mathrm{NS}} / \rho$, assume a NS mass of $1.4 M_{\odot}$, and take $\Delta t_{\mathrm{NS}}=7.5 \mathrm{~s}$. This time scale corresponds to the SN 1987A energy-loss argument, assuming that at most half of the total energy goes into KK gravitons. Finally we need to divide by $4 \pi d^{2}$ with $d=3.4 \mathrm{kpc}$ the distance to Cas A. We further need a factor 2 for two decay photons per graviton. We also assume that for every decaying $\mathrm{KK}$ graviton we have $E_{\gamma}=\omega / 2$, i.e. we ignore the narrow energy distribution of the decay photons from non-relativistic gravitons. Collecting all factors we predict an EGRET flux at Earth of

$$
\begin{aligned}
\Phi_{E_{\gamma}>E_{0}} & =\frac{\Omega_{n} R^{n}}{(2 \pi)^{2}} \frac{V_{\mathrm{NS}} \Delta t_{\mathrm{NS}} \gamma}{4 \pi d^{2}} \\
& \times 2 \int_{2 E_{0}}^{\infty} d \omega S(-\omega) \int_{0}^{\omega} d m \omega^{n+3} G_{n+3}(m / \omega)
\end{aligned}
$$

where $E_{0}=100 \mathrm{MeV}$. The $\int d m$ integral is explicitly $\omega^{n+4} h_{n}$ with

$$
h_{n}=\frac{5 n\left(278+223 n+48 n^{2}+3 n^{3}\right) \sqrt{\pi} \Gamma\left(\frac{n}{2}\right)}{384 \Gamma\left(\frac{n+11}{2}\right)} .
$$

Therefore, we have

$$
\Phi_{E_{\gamma}>E_{0}}=\Phi_{0} h_{n} \Omega_{n}(R T)^{n} \phi_{n}\left(2 E_{0} / T\right)
$$

where

$$
\phi_{n}(u)=\int_{u}^{\infty} d x \frac{x^{2+n}}{1+e^{x}}
$$

and

$$
\begin{aligned}
\Phi_{0} & =\frac{1}{(2 \pi)^{2}} \frac{V_{\mathrm{NS}} \Delta t_{\mathrm{NS}} \gamma 4 S_{0} T^{3}}{4 \pi d^{2}} \\
& =7.1 \times 10^{-24} \mathrm{~cm}^{-2} \mathrm{~s}^{-1} T_{30}^{11 / 2} \rho_{3} .
\end{aligned}
$$

We given explicit values for $\left(\Omega_{n} h_{n}\right)^{1 / n}$ in Table

Comparing with Eq. (45) then gives us the limits on $R$ and $\bar{M}_{4+n}$ shown in Tables $[\mathrm{V}$ ] and $[\mathrm{VI}$ respectively, for $T=30 \mathrm{MeV}$. In contrast to the previous results they now depend rather sensitively on the $\mathrm{SN}$ core temperature. Reducing $T$ to $20 \mathrm{MeV}$ degrades the $R$-limit for $n=2$ by about a factor of 10 , for $n=7$ still by about a factor of 2 . The $\bar{M}_{4+n}$ limits are somewhat less sensitive. Changing from 30 to $20 \mathrm{MeV}$ degrades the $n=2$ limit by about a factor of 3.5, the $n=7$ limit by about a factor of 2 . Overall the Cas A limits are comparable to those from all past SNe.

\section{Gamma Radiation from Neutron Stars}

A large fraction of the KK gravitons emitted by a SN core are gravitationally retained so that every NS would be embedded in a halo of these particles. Therefore, NSs would be bright sources of $100 \mathrm{MeV} \gamma$-rays visible to EGRET. The flux of decay photons expected from a NS can be written in a way which is very similar to Eq. (47), except that the integral over $d m$ is changed so that only the trapped gravitons are counted

$$
\Phi_{E_{\gamma}>E_{0}}=\Phi_{0}^{*} \Omega_{n}(R T)^{n} I_{n}\left(2 E_{0} / T\right),
$$


where

$$
\Phi_{0}^{*}=8.1 \times 10^{-23} \mathrm{~cm}^{-2} \mathrm{~s}^{-1} T_{30}^{11 / 2} \rho_{3} d_{\mathrm{kpc}}^{-2} .
$$

The function $I_{n}(u)$ is defined as

$$
\begin{aligned}
I_{n}(u) & =\frac{1}{3} \int_{0}^{1} r^{2} d r \int_{u}^{\infty} d x \frac{x^{n+2}}{1+e^{x}} \\
& \times \int_{0}^{1-U(r)} d \mu G_{n+3}(\mu) .
\end{aligned}
$$

In Table III we give $I_{n}$ for $n=1-7$ and $T=30 \mathrm{MeV}$.

Comparing this flux prediction with the EGRET pointsource limit Eq. (45) for various old NSs then allows one to derive limits on $R$. In our previous paper [12] we used several old NSs for this argument. The most restrictive limits obtained from the nearest neutron star RX J185634-3754 that was taken to be at a distance of $60 \mathrm{pc}$ 20 and from PSR J0953+0755 at 120 pc 21. However, the distance of RX J185634-3754 has recently been adjusted upwards to $117 \pm 12$ pc [22], so that the distance is now exactly the same as for PSR J0953+0755. This in turn means that the derived limits on $R$ from the gamma-ray luminosity is exactly the same for the two stars. The limits on $R$ and $\bar{M}_{4+n}$ thus derived are tabulated in Tables $\nabla$ and VI respectively.

The flux prediction of Eq. (49) scales with the SN core temperature as $T^{11 / 2}$, just as for the Cas A argument. Therefore, the limits are equally sensitive to $T$ as discussed in the previous section.

\section{E. Neutron-Star Excess Heat}

\section{Decay of KK gravitons}

Even more stringent constraints obtain from considering the heating of old NSs by the trapped cloud of KK gravitons surrounding them [12]. When a graviton decays outside the NS, the fraction of the decay photons hitting the surface of the star is $\frac{1}{2} \sqrt{d^{2}-R_{\mathrm{NS}}^{2}} / d$, where $d$ is the distance of the graviton from the center of the star, and $R_{\mathrm{NS}}$ is the NS radius.

We proceed in the following way to calculate the rms distance of a particle in the graviton cloud from the center of the star $\left\langle d^{2}\right\rangle^{1 / 2}$. First we calculate the average total energy $E=\frac{1}{2} v^{2}+U(r)$ for the trapped gravitons. We then assume for simplicity that all gravitons are on purely radial orbits. Given the level of approximations entering this calculation this is certainly justified. By solving the equation of motion for a graviton with energy $E$ on a radial orbit we get $\left\langle d^{2}\right\rangle^{1 / 2}$, and from this we can calculate the fraction, $\mathcal{F}$, of the total KK-produced photon flux absorbed by the NS. In doing this we assume that all gravitons decay when they are at a distance of exactly $\left\langle d^{2}\right\rangle^{1 / 2}$. Numerical values for $\left\langle d^{2}\right\rangle^{1 / 2}$ and $\mathcal{F}$ are shown in Table IV for $T=30 \mathrm{MeV}$.
TABLE III: Values of the parameter $J_{n}$, and the functions $\phi_{n}\left(2 E_{0} / T\right)$ and $I_{n}\left(2 E_{0} / T\right)$, taken for $T=30 \mathrm{MeV}$ and $E_{0}=$ $100 \mathrm{MeV}$.

\begin{tabular}{llll}
\hline \hline$n$ & $\phi_{n}\left(2 E_{0} / T\right)$ & $I_{n}\left(2 E_{0} / T\right)$ & $J_{n}$ \\
1 & 0.605 & $7.56 \times 10^{-3}$ & 0.292 \\
2 & 4.933 & 0.0443 & 1.061 \\
3 & 41.42 & 0.274 & 4.731 \\
4 & 360.2 & 1.797 & 25.05 \\
5 & $3.27 \times 10^{3}$ & 12.48 & 153.8 \\
6 & $3.11 \times 10^{4}$ & 92.17 & 1074.5 \\
7 & $3.13 \times 10^{5}$ & 727.3 & 8428.2 \\
\hline \hline
\end{tabular}

TABLE IV: Values of $\left\langle d^{2}\right\rangle^{1 / 2}, \mathcal{F}, t_{\text {inside }}$, and the reabsorption rate $\Gamma_{n}$.

\begin{tabular}{lllll}
\hline \hline$n$ & $\left\langle d^{2}\right\rangle^{1 / 2}$ & $\mathcal{F}$ & $t_{\text {inside }}$ & $\Gamma_{n}\left[\mathrm{~s}^{-1}\right]$ \\
1 & 1.405 & 0.149 & 0.269 & $1.22 \times 10^{-16}$ \\
2 & 1.372 & 0.158 & 0.279 & $2.06 \times 10^{-17}$ \\
3 & 1.352 & 0.164 & 0.287 & $7.18 \times 10^{-18}$ \\
4 & 1.320 & 0.174 & 0.297 & $3.44 \times 10^{-18}$ \\
5 & 1.291 & 0.184 & 0.308 & $1.94 \times 10^{-18}$ \\
6 & 1.262 & 0.195 & 0.319 & $1.21 \times 10^{-18}$ \\
7 & 1.236 & 0.206 & 0.330 & $8.14 \times 10^{-19}$ \\
\hline \hline
\end{tabular}

Having calculated the fraction of the total flux absorbed by the star, we can calculate the total energy per unit time absorbed by the star, $L$, as

$$
\begin{aligned}
L= & \frac{\Omega_{n} R^{n}}{(2 \pi)^{2}} V_{\mathrm{NS}} \Delta t_{\mathrm{NS}} \gamma \mathcal{F} \frac{1}{3} \int_{0}^{1} r^{2} d r \\
& \times \int_{0}^{\infty} d \omega \omega^{n+5} S(-\omega) \int_{0}^{1-U(r)} d \mu G_{n+3}(\mu) \\
= & 6.17 \times 10^{-17} L_{\odot} \Omega_{n}(R T)^{n} \mathcal{F} J_{n} \rho_{3} T_{30}^{13 / 2} .
\end{aligned}
$$

Values for the parameter

$$
\begin{aligned}
J_{n} & =\frac{1}{3} \int_{0}^{1} r^{2} d r \int_{0}^{\infty} d x \frac{x^{n+3}}{1+e^{x}} \\
& \times \int_{0}^{1-U(r)} d \mu G_{n+3}(\mu)
\end{aligned}
$$

are shown Table III

The thermal cooling time scale of an isolated neutron star is of order $10^{5}-10^{6} \mathrm{yr}$. The neutron star PSR $\mathrm{J} 0953+0755$ has an estimated age of $17 \times 10^{6} \mathrm{yr}$, and HST observations indicate that it has a total luminosity of roughly $10^{-5} L_{\odot}[23]$. Comparing this to the total energy transferred to the star from KK graviton decay, Eq. (55), yields an upper limit on $R$. In Tables $\nabla$ and VI we have tabulated limits on $R$ and $\bar{M}_{4+n}$ derived from the maximum energy transfer per unit time to the neutron star $L_{\max }=10^{-5} L_{\odot}$. As we discussed in Ref. [12], this limit is far stronger than other astrophysical limits on $R$. For $n<5$ it is stronger than any current laboratory limits. 
TABLE V: Upper limits on the compactification radius $R$ (in meters) from our arguments.

\begin{tabular}{|c|c|c|c|c|c|c|c|}
\hline$n$ & 1 & 2 & 3 & 4 & 5 & 6 & 7 \\
\hline \multicolumn{8}{|l|}{ Neutrino Signal } \\
\hline SN 1987A & $4.9 \times 10^{2}$ & $0.96 \times 10^{-6}$ & $1.14 \times 10^{-9}$ & $3.82 \times 10^{-11}$ & $4.85 \times 10^{-12}$ & $1.21 \times 10^{-12}$ & $4.42 \times 10^{-13}$ \\
\hline \multicolumn{8}{|l|}{ EGRET $\gamma$-ray limits } \\
\hline All cosmic SNe & 4.9 & $0.96 \times 10^{-7}$ & $2.47 \times 10^{-10}$ & $1.21 \times 10^{-11}$ & $1.93 \times 10^{-12}$ & $5.6 \times 10^{-13}$ & $2.29 \times 10^{-13}$ \\
\hline Cas A & $4.1 \times 10^{2}$ & $3.61 \times 10^{-7}$ & $3.95 \times 10^{-10}$ & $1.37 \times 10^{-11}$ & $1.86 \times 10^{-12}$ & $5.0 \times 10^{-13}$ & $1.94 \times 10^{-13}$ \\
\hline $\left.\begin{array}{l}\text { PSR J0953+0755 } \\
\text { RX J185635-3754 }\end{array}\right\}$ & 1.935 & $2.63 \times 10^{-8}$ & $7.17 \times 10^{-11}$ & $3.91 \times 10^{-12}$ & $6.99 \times 10^{-13}$ & $2.24 \times 10^{-13}$ & $9.98 \times 10^{-14}$ \\
\hline \multicolumn{8}{|c|}{ Neutron-star excess heat } \\
\hline PSR J0952+0755 & $4.44 \times 10^{-5}$ & $1.55 \times 10^{-10}$ & $2.58 \times 10^{-12}$ & $3.36 \times 10^{-13}$ & $9.95 \times 10^{-14}$ & $4.41 \times 10^{-14}$ & $2.46 \times 10^{-14}$ \\
\hline
\end{tabular}

TABLE VI: Lower limits on the fundamental energy scale $\bar{M}_{4+n}$ (in TeV) corresponding to the $R$-limits of Table $\mathrm{V}$ Multiply limits on $\bar{M}_{4+n}$ by the factor $M / \bar{M}_{n+4}$ given in the second row to obtain limits on the parameter $M$ used in our previous papers [11, 12].

\begin{tabular}{|c|c|c|c|c|c|c|c|}
\hline$n$ & 1 & 2 & 3 & 4 & 5 & 6 & 7 \\
\hline$M / \bar{M}_{n+4}$ & 2.32 & 2.98 & 3.46 & 3.82 & 4.10 & 4.32 & 4.51 \\
\hline \multicolumn{8}{|l|}{ Neutrino Signal } \\
\hline SN 1987A & $7.4 \times 10^{2}$ & 8.9 & 0.66 & $1.18 \times 10^{-1}$ & $3.5 \times 10^{-2}$ & $1.44 \times 10^{-2}$ & $7.2 \times 10^{-3}$ \\
\hline \multicolumn{8}{|l|}{ EGRET $\gamma$-ray limits } \\
\hline All cosmic SNe & $3.4 \times 10^{3}$ & 28. & 1.65 & $2.54 \times 10^{-1}$ & $6.8 \times 10^{-2}$ & $2.56 \times 10^{-2}$ & $1.21 \times 10^{-2}$ \\
\hline Cas A & $7.7 \times 10^{2}$ & 14.5 & 1.24 & $2.34 \times 10^{-1}$ & $7.0 \times 10^{-2}$ & $2.80 \times 10^{-2}$ & $1.37 \times 10^{-2}$ \\
\hline $\left.\begin{array}{l}\text { PSR J0953+0755 } \\
\text { RX J185635-3754 }\end{array}\right\}$ & $4.58 \times 10^{3}$ & 54.0 & 3.46 & 0.54 & 0.14 & $5.10 \times 10^{-2}$ & $2.30 \times 10^{-2}$ \\
\hline \multicolumn{8}{|l|}{ Neutron-star excess heat } \\
\hline PSR J0952+0755 & $1.61 \times 10^{5}$ & $7.01 \times 10^{2}$ & 25.5 & 2.77 & 0.57 & 0.17 & $6.84 \times 10^{-2}$ \\
\hline
\end{tabular}

The predicted NS heating rate of Eq. (55) scales with the SN core temperature as $T^{13 / 2}$, slightly steeper than for the previous arguments. The $T$-sensitivity of the limits on $R$ and $\bar{M}_{4+n}$ is almost the same as before.

\section{Reabsorption in the neutron star}

KK gravitons in the trapped cloud will be inside the NS on part of their trajectory. Assuming, as before, that all gravitons move on radial orbits we have calculated the fraction of time an average graviton spends inside the NS, $t_{\text {inside. }}$ While the graviton is inside the neutron star it can potentially be reabsorbed by the nuclear medium. Using Eq. (20) we find the following absorption rates for average gravitons

$$
\Gamma_{\text {reabsorption }}=\Gamma_{n} \rho_{3}^{2} T_{30}^{-1 / 2}
$$

where values of $\Gamma_{n}$ are tabulated in Table IV Comparing this reabsorption rate to the age of the two NSs RX J185634-3754 (1.6 × 10 $\left.{ }^{13} \mathrm{~s}\right)$ and PSR J0953+0755 $\left(5.4 \times 10^{14} \mathrm{~s}\right)$ we see that reabsorption in the medium is at most a moderate effect.

\section{CONCLUSIONS}

We have systematically revisited the constraints on KK graviton emission by SN cores and neutron stars that have been discussed in the literature. We have paid close attention to the scaling with the number $n$ of extra dimensions and to the conventions concerning the relationship between the radius $R$ of the large extra dimensions and the fundamental energy scale, the $4+n$ dimensional effective Planck mass $\bar{M}_{4+n}$. Our limits are summarized in Tables $\nabla$ and VI] For $n=2$ and 3 the limits based on EGRET observations are slightly less restrictive than stated in our previous papers, largely because we use a somewhat smaller emission rate from a SN core, and because the estimated distance to RX J185635-3754 has increased by a factor of 2 . However, the bound from neutron star excess heat is in fact slightly more restrictive than stated in our previous paper. The reason is that the average distance of a graviton from the neutron star center is somewhat lower in the present calculation, compared to what was used in our previous work.

The main theoretical new ingredient of our work is a new approach to calculating the emission rate. For lowenergy gravitational bremsstrahlung the emission rate can be calculated directly from the classical formula in conjunction with the universal detailed-balancing property of the medium's dynamical structure function. This calculation agrees with previous works to the specified 
order of precision, but is vastly simpler and clearly illuminates the nature of the used approximation. The KK graviton emission rate by a $\mathrm{SN}$ core can not be calculated to better than a factor of a few. However, this uncertainty hardly affects the limits, especially for large $n$, because the limiting value of $R$ is proportional to the $n$-th root of the limiting emission rate.

Another benefit of our derivation is that it produces directly the absorption rate. It is found to be so small that the gravitationally trapped KK gravitons are not reabsorbed too quickly to invalidate our previous arguments.

\section{Acknowledgments}

In Munich, this work was partly supported by the Deutsche Forschungsgemeinschaft under grant SFB 375 and the ESF network Neutrino Astrophysics. G.R. thanks the Scuola Internazionale Superiore di Studi Avanzati (SISSA), Trieste, for hospitality during a visit where this work was begun. S.H. thanks the MPI für Physik for hospitality while this paper was finished.
[1] N. Arkani-Hamed, S. Dimopoulos and G. Dvali, "The hierarchy problem and new dimensions at a millimeter," Phys. Lett. B 429, 263 (1998) hep-ph/9803315.

[2] I. Antoniadis, N. Arkani-Hamed, S. Dimopoulos and G. Dvali, "New dimensions at a millimeter to a Fermi and superstrings at a TeV," Phys. Lett. B 436, 257 (1998) hep-ph/9804398.

[3] N. Arkani-Hamed, S. Dimopoulos and G. Dvali, "Phenomenology, astrophysics and cosmology of theories with sub-millimeter dimensions and $\mathrm{TeV}$ scale quantum gravity," Phys. Rev. D 59, 086004 (1999) hep-ph/9807344.

[4] T. Han, J. D. Lykken and R. Zhang, "On Kaluza-Klein states from large extra dimensions, Phys. Rev. D 59, 105006 (1999) hep-ph/9811350.

[5] G. F. Giudice, R. Rattazzi and J. D. Wells, "Quantum gravity and extra dimensions at high-energy colliders," Nucl. Phys. B 544, 3 (1999) hep-ph/9811291.

[6] J. Hewett and J. March-Russell, "Extra Dimensions," in: K. Hagiwara et al. (Particle Data Group), "The Review of Particle Physics," Phys. Rev. D 66, 010001 (2002).

[7] S. Cullen and M. Perelstein, "SN1987A constraints on large compact dimensions," Phys. Rev. Lett. 83, 268 (1999) hep-ph/9903422.

[8] V. Barger, T. Han, C. Kao and R. J. Zhang, "Astrophysical constraints on large extra dimensions," Phys. Lett. B 461, 34 (1999) hep-ph/9905474.

[9] C. Hanhart, D. R. Phillips, S. Reddy and M. J. Savage, "Extra dimensions, SN 1987A, and nucleon nucleon scattering data," Nucl. Phys. B 595, 335 (2001) nucl-th/0007016.

[10] C. Hanhart, J. A. Pons, D. R. Phillips and S. Reddy, "The likelihood of GODs' existence: Improving the SN 1987A constraint on the size of large compact dimensions," Phys. Lett. B 509, 1 (2001) astro-ph/0102063.

[11] S. Hannestad and G. Raffelt, "New supernova limit on large extra dimensions: Bounds on Kaluza-Klein graviton production," Phys. Rev. Lett. 87, 051301 (2001) hep-ph/0103201.
[12] S. Hannestad and G. G. Raffelt, "Stringent neutron-star limits on large extra dimensions," Phys. Rev. Lett. 88, 071301 (2002) hep-ph/0110067.

[13] S. Weinberg, "Gravitation and Cosmology" (Wiley, New York, 1972).

[14] G. Raffelt and D. Seckel, "A selfconsistent approach to neutral current processes in supernova cores," Phys. Rev. D 52, 1780 (1995) astro-ph/9312019.

[15] H. T. Janka, W. Keil, G. Raffelt and D. Seckel, "Nucleon spin fluctuations and the supernova emission of neutrinos and axions," Phys. Rev. Lett. 76, 2621 (1996) astro-ph/9507023.

[16] G. G. Raffelt, "Stars As Laboratories For Fundamental Physics," (University of Chicago Press, 1996).

[17] G. G. Raffelt, "Particle physics from stars," Ann. Rev. Nucl. Part. Sci. 49, 163 (1999) hep-ph/9903472.

[18] T. Han, J. D. Lykken and R. J. Zhang, "On KaluzaKlein states from large extra dimensions," Phys. Rev. D 59, 105006 (1999) hep-ph/9811350.

[19] D. A. Kniffen et al., "EGRET Observations of the high latitude diffuse radiation," Astron. Astrophys. Suppl. Ser. 120, 615 (1996).

[20] F. M. Walter, "The proper motion, parallax, and origin of the isolated neutron star RX J185635-3754," Astrophys. J. 549, 433 (2001). astro-ph/0009031.

[21] T. M. Tauris et al., "Discovery of PSR J0108-1431: The closest known neutron star?," Astrophys. J. Lett. 428, L53 (1994).

[22] F. M. Walter and J. M. Lattimer, "A revised parallax and its implications for RX J185635-3754," Astrophys. J. Lett. 576, L145 (2002).

[23] M. B. Larson and B. Link, "Superfluid friction and latetime thermal evolution of neutron stars," Astrophys. J. 521, 271 (1999) astro-ph/9810441. 


\section{Erratum "Supernova and neutron-star limits on large extra dimensions reexamined"}

(Dated: 13 October 2003)

TABLE V: Upper limits on the compactification radius $R$ (in meters) from our arguments.

\begin{tabular}{|c|c|c|c|c|c|c|c|}
\hline$n$ & 1 & 2 & 3 & 4 & 5 & 6 & 7 \\
\hline \multicolumn{8}{|l|}{ Neutrino Signal } \\
\hline SN 1987A & $4.9 \times 10^{2}$ & $0.96 \times 10^{-6}$ & $1.14 \times 10^{-9}$ & $3.82 \times 10^{-11}$ & $4.85 \times 10^{-12}$ & $1.21 \times 10^{-12}$ & $4.42 \times 10^{-13}$ \\
\hline \multicolumn{8}{|l|}{ EGRET $\gamma$-ray limits } \\
\hline Cas A & $4.1 \times 10^{2}$ & $3.61 \times 10^{-7}$ & $3.95 \times 10^{-10}$ & $1.37 \times 10^{-11}$ & $1.86 \times 10^{-12}$ & $5.0 \times 10^{-13}$ & $1.94 \times 10^{-13}$ \\
\hline $\left.\begin{array}{l}\text { PSR J0953+0755 } \\
\text { RX J185635-3754 }\end{array}\right\}$ & 7.36 & $5.13 \times 10^{-8}$ & $1.12 \times 10^{-10}$ & $5.46 \times 10^{-12}$ & $9.13 \times 10^{-13}$ & $2.80 \times 10^{-13}$ & $1.21 \times 10^{-13}$ \\
\hline
\end{tabular}

TABLE VI: Lower limits on the fundamental energy scale $\bar{M}_{4+n}$ (in TeV) corresponding to the $R$-limits of Table $\nabla$

\begin{tabular}{|c|c|c|c|c|c|c|c|}
\hline $\bar{n}$ & 1 & 2 & 3 & 4 & 5 & 6 & 7 \\
\hline$M / \bar{M}_{n+4}$ & 2.32 & 2.98 & 3.46 & 3.82 & 4.10 & 4.32 & 4.51 \\
\hline \multicolumn{8}{|l|}{ Neutrino Signal } \\
\hline SN 1987A & $7.4 \times 10^{2}$ & 8.9 & 0.66 & $1.18 \times 10^{-1}$ & $3.5 \times 10^{-2}$ & $1.44 \times 10^{-2}$ & $7.2 \times 10^{-3}$ \\
\hline All cosmic SNe & $3.4 \times 10^{3}$ & 28. & 1.65 & $2.54 \times 10^{-1}$ & $6.8 \times 10^{-2}$ & $2.56 \times 10^{-2}$ & $1.21 \times 10^{-2}$ \\
\hline Cas A & $7.7 \times 10^{2}$ & 14.5 & 1.24 & $2.34 \times 10^{-1}$ & $7.0 \times 10^{-2}$ & $2.80 \times 10^{-2}$ & $1.37 \times 10^{-2}$ \\
\hline $\left.\begin{array}{l}\text { PSR J0953+0755 } \\
\text { RX J185635-3754 }\end{array}\right\}$ & $2.93 \times 10^{3}$ & 38.6 & 2.65 & 0.43 & 0.116 & $4.31 \times 10^{-2}$ & $1.98 \times 10^{-2}$ \\
\hline \multicolumn{8}{|l|}{ Neutron-star excess heat } \\
\hline PSR J0952+0755 & $1.61 \times 10^{5}$ & $7.01 \times 10^{2}$ & 25.5 & 2.77 & 0.57 & 0.17 & $6.84 \times 10^{-2}$ \\
\hline
\end{tabular}

In our paper "Supernova limits on large extra dimensions reexamined" [Phys. Rev. D 67, 125008 (2003)] a small error has occurred. We have mistakenly used the old distance of $60 \mathrm{pc}$ rather than the new value of $117 \mathrm{pc}$ for the nearby neutron star RX J185635-3754. Therefore, the corresponding limits on $R$ are degraded by a factor $(117 / 60)^{2 / n}$ while those on $\bar{M}_{4+n}$ need a factor $(60 / 117)^{2 /(2+n)}$. Below we reproduce Tables V and VI with the corrected limits in boldface. We thank G. Sigl for drawing our attention to this error.

Note that these limits can be significantly improved if one uses all neutron stars in the galactic bulge as a source rather than only the single nearby stars RX J1856353754 and PSR J0953+0755. The loss caused by the greater distance is significantly overcompensated by the large number of neutron stars. [M. Casse, J. Paul, G. Bertone and G. Sigl, "Gamma rays from the galactic bulge and large extra dimensions", hep-ph/0309173.] 